\title{
ColorSEM: Livetime Quantitative X-ray Mapping in an Integrated EDS Workflow
}

Petr Wandrol $^{1}$, Tomáš Tůma ${ }^{1}$, Jan Klusáček ${ }^{1}$, Jiří Petřek ${ }^{1}$, Zbyněk Vraštil ${ }^{1}$ and Chris Stephens ${ }^{2}$

${ }^{1}$ Thermo Fisher Scientific, Brno, Jihomoravsky kraj, Czech Republic, ${ }^{2}$ Thermo Fisher Scientific, East Grinstead, England, United Kingdom

Energy dispersive X-ray spectroscopy (EDS) is the most widely used analytical technique for SEM, and is used in a broad and diverse range of applications. Although commercially available for over 50 years, recent years have seen an acceleration in the rate of EDS development, which has in turn led to the use of EDS in a number of new applications areas, such as $\mathrm{Li}$ analysis in batteries, thin layer analysis in semiconductors and section reconstruction in biological sciences. For hardware, large area silicon drift detectors (SDDs) combined with low absorption windows and advanced digital processing enable excellent resolutions and low $\mathrm{kV}$ performance, even at very high throughputs. For software, highly accurate standardless quantification routines, increased use of automation and big-data statistical techniques enables mapping over very large areas in practical timescales.

ColorSEM represents the latest evolution of SEM EDS technology [1], and is built upon the tightest possible integration of the SEM and EDS to enable live time chemical imaging. This sits alongside traditional EDS functionality, such as Point and ID, Line Scan and elemental X-ray mapping to provide the fastest time to data. This paper describes the ColorSEM data processing workflow, including EDS detector design, pulse processing, standardless quantification and graphical user interface, and how it can be used to acquire EDS maps at real-time scanning rates.

SEM scan generators are designed for fast and accurate image acquisition; using the same engine for EDS mapping allows for dwell times as short as $50 \mathrm{ns,} \mathrm{which} \mathrm{reduces} \mathrm{electron} \mathrm{dose} \mathrm{and} \mathrm{beam} \mathrm{damage} \mathrm{for}$ sensitive samples. However, maps acquired at such dwell times have sparse data even at high count rate. For example, a $50 \mathrm{~ns}$ dwell time would require an output count rate of 20 million cps to average one x-ray per pixel in a single pass. Up to now SEM users were required to use either high probe currents, large area detectors or long acquisition times in order to avoid sacrificing X-ray map resolution. ColorSEM solves this by using the multiple signal types available in the SEM, segmenting the SE and BSE images using machine vision algorithms based on the contrast between features and objects in the image. EDS events from each segment, or superpixel, are quantified through an optimized standardless quantification routine and assigned a color representative of the relative prevalence of the chemical elements present. Once the number of counts in a given superpixel increases beyond a confidence threshold, the superpixel divides into smaller segment and the process is repeated. The superpixel size continually decreases with time, capturing small composition changes not identified in regular SE or BSE images.

The ColorSEM standardless quantification routine includes elemental ID, standardless peak fitting with peak deconvolution by linear least squared fit, and Phi-rho-Z (PROZA) matrix correction [2]. Figure 1 shows $\mathrm{MoO}_{3}$ and $\mathrm{Sb}_{2} \mathrm{~S}_{5}$ composite, acquired at $20 \mathrm{kV}, 3.2 \mathrm{nA}$ beam current and 80 seconds livetime using a Thermo Scientific Prisma E SEM equipped with a $30 \mathrm{~mm}^{2}$ SDD EDS detector. Gross count mapping provides false information, due to the strong overlap between the $\mathrm{S} \mathrm{K} \alpha$ and Mo L $\alpha$ peaks, which are correctly resolved in the ColorSEM live quantification mapping.

ColorSEM is always on, acquiring and processing EDS data for as long as the electron beam is on. Redundancies associated with traditional EDS acquisition via external beam control are eliminated, providing a much shorter pathway to quantitative data. Since ColorSEM is a fully integrated system, it is 
also possible to provide complementary information from other modalities simultaneously during acquisition, such as cathodoluminescence (CL). Figure 2 shows the variability of data that can be obtained by the SEM with an integrated EDS and CL. ColorSEM quant mapping ensures proper identification of zircons and phosphate particle in the mineral sample. Zonation of zircons can be further visualized by the integrated CL detector providing additional details about their composition and crystal growth. All the correlative information can be acquired within few minutes using the fully integrated system.

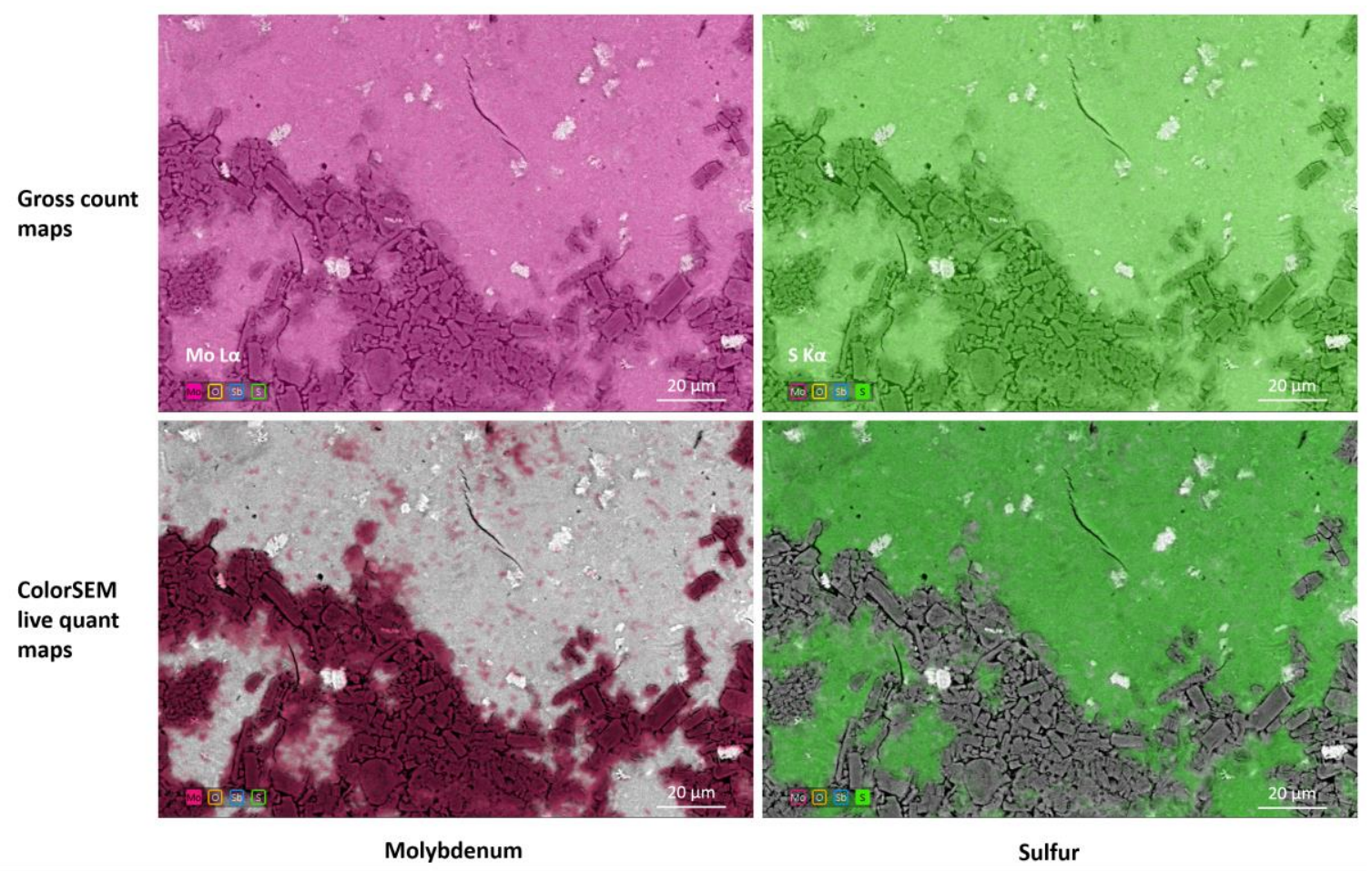

Figure 1. Comparison of gross count and quant elemental maps of a MoO3 and Sb2S5 composite. Molybdenum and Sulfur peaks overlap each other (Mo L $\alpha=2.292 \mathrm{keV}$ and S K $\alpha=2.309 \mathrm{keV}$ ). While gross count mapping can't resolve these two phases, quant mapping solves this issue by on the fly deconvolution and matrix corrections. 

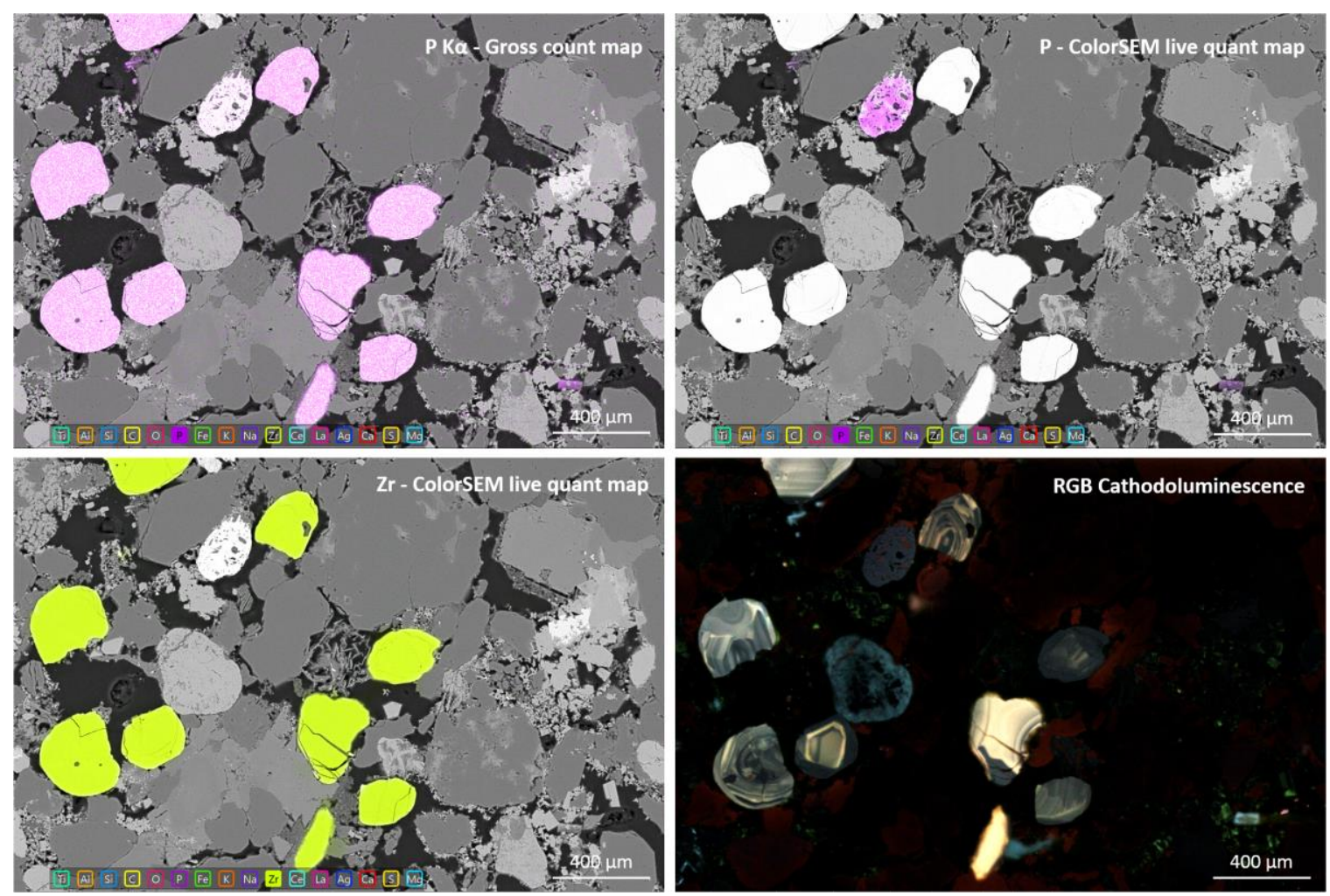

Figure 2. Mineral sample containing Zircons and Monazite phosphate particle next to each other. Gross count maps $\mathrm{P} \mathrm{K} \alpha$ shows misidentification of zircons (identified as phosphate particles). ColorSEM live quant mapping solves $\mathrm{Zr}$ and $\mathrm{P}$ overlap (P K $\alpha=2.010 \mathrm{keV}$ and $\mathrm{Zr} \mathrm{L} \alpha=2.044 \mathrm{keV}$ ) and distinguishes between phosphate particle and Zircons. Zonation of zircons is visualized further by the integrated CL detector providing additional details about their composition and crystal growth.

\section{References}

[1] Vesseur E. J., Microsc. Microanal. 25 (Suppl 2), 2019, p. 562

[2] Bastin G. F., Heijligers H. J. M., van Loo F. J. J., Scanning Vol. 8, 1986, p. 45 